collar for 3 to 6 weeks, administration of drugs such as anti-inflammatory agents, analgesics, muscle relaxants and sedatives, nerve blocking and motorized intermittent traction. All of the cases were treated in the outpatient clinic, and at their first consultation each patient was given a printed matter on which directions and explanations about therapy are noted.

In the present report, 142 cases were analysed, who came to our clinic within one week following injuries and were treated under the principle mentioned above.

Conclusions are as follows:

1) The result of the treatment was good in 73 , fair in 56 and poor in 13, after the follow up of 2 to 8 months.

2) X-ray examinations of the neck were performed in 118 cases and abnormalities were found in 56 out of them. Patients having abnormal X-ray findings showed poor results, compared with those having no abnormalities.

3) All patients were classified into four groups of type according to their signs and symptoms. They are the upper cervical type, lower cervical type, vertebral artery type and mixed type. The mixed type showed poorer prognosis than the other types.

4) From the fact that cases injured by rear end collision show the mixed type syndrome more frequently than cases of the other kinds of accident, it is presumed that hyperextension injury may cause severe cervical syndrome more easily.

\title{
189. The Surgical Treatment to the Neck Injuries
}

\author{
Noboru NAKANO \\ Nakano Orthopaedic Institution
}

The neck injuries by car wreck has increased recently in Japan but neck injuries is not new as they considered and there are nothing different between symptoms by car wreck and other causes, which are headache, pain at neck, numbness at upper extremity, weakness of grasp power, etc. Anterior interbody fusion was done to 65 patients who had those symptoms which was 17 at one level, 38 at two level, 9 at three level and 1 at four level. 22 cases, about only $33 \%$ out of 65 cases were caused by car wreck and all of those patients had neck traction just after injury which had not to be done.

Result was investigated on 59 cases which was excellent in 34 , good in 23 , fair in 2 and there was no poor case.

If proper treatment as spica cast is applied at early stage, number of surgery to the patient with neck injuries will be decreased.

Also patient should not be gather in same room because they exchanges their symptoms each other. 